\title{
Evaluación del estado nutricio de los pacientes adultos mayores en periodo postoperatorio utilizando el instrumento Mini Nutritional Assessment (MNA) en Yucatán
}

\author{
Ana Ligia Gutiérrez-Solis*, Rodrigo García-Pérez, Sarahí Herrera-Escalante, \\ Jocelyne de la Cruz-Acosta y Roberto Lugo \\ Unidad de Investigación, Hospital Regional de Alta Especialidad de la Península de Yucatán. Mérida, Yuc., México
}

\begin{abstract}
Background: During the nutritional assessment of elderly patients, it is important to take into account the physical changes of aging. It is well known that hospitalized patients are more vulnerable to develop malnutrition. The Mini Nutritional Assessment (MNA) questionnaire allows the adequate classification of the nutritional status of these patients. In Yucatan, there are no reports on the nutritional status among this group of patients. Therefore, the aim of this study was to assess nutritional status of elderly patients in this referral hospital using the MNA questionnaire. Methods: A total of 100 hospitalized patients older than 60 years were evaluated from February to May 2019. The MNA questionnaire was applied, anthropometric measurements were collected and laboratory tests were done. Results: $55 \%$ were female and $45 \%$ were male. According to MNA, 53\% of the population was classified at risk of malnutrition, $31 \%$ with malnutrition and $26 \%$ as having a normal nutritional status. Conclusions: The evaluation of the nutritional status among elderly hospitalized patients using the MNA showed that half of the population were at risk of malnutrition. Early recognition of malnutrition and adequate intervention among hospitalized patients can prevent later complications in the treatment and convalescence period. (Hosp Med Clin Manag. 2019;12:113-9) Corresponding author: Ana Ligia Gutiérrez-Solis, ganaligia@gmail.com
\end{abstract}

Key words: Nutritional status. Elderly. Mini Nutritional Assessment.

\section{RESUMEN}

Antecedentes: Los pacientes adultos mayores en periodo postoperatorio pueden presentar mayor vulnerabilidad a desarrollar cuadros de malnutrición, por lo que es importante considerarlo al momento de realizar su valoración nutricional. El cuestionario Mini Nutritional Assessment (MNA) permite clasificar adecuadamente el estado nutricio de estos pacientes. En Yucatán no existen reportes del estado nutricio de estos pacientes en el periodo postoperatorio. El objetivo de este estudio fue evaluar el estado nutricional en adultos mayores en periodo postoperatorio utilizando el MNA. Metodología: Fueron evaluados 100 pacientes mayores de 60 años en periodo postoperatorio, intervenidos

\section{Correspondencia:}

*Ana Ligia Gutiérrez-Solis

E-mail: ganaligia@gmail.com
Date of reception: 23-05-2019

Date of acceptance: 29-08-2019

DOI: 10.24875/HMCM.19000211 
en el hospital durante febrero a mayo del 2019. Se aplicó el cuestionario MNA, realizando una serie de preguntas, estudios de laboratorio y medidas antropométricas. Resultados: El 55\% de los pacientes fueron de sexo femenino y $45 \%$ de sexo masculino. El 53\% presentaron riesgo de malnutrición, el 31\% malnutrición y el 16\% estado nutricional normal. Conclusión: La evaluación del estado nutricional utilizando el MNA indicó que más de la mitad de la población adulta mayor hospitalizada se encuentra en riesgo de malnutrición. El reconocimiento temprano de la malnutrición y la intervención adecuada en pacientes hospitalizados pueden prevenir complicaciones posteriores en el tratamiento y lograr la mejoría de estos pacientes.

Palabras clave: Estado nutricional. Adulto mayor. Postoperatorio. Mini Nutritional Assessment.

\section{INTRODUCCIÓN}

En la clínica se define como malnutrición al déficit de nutrientes que lleva a la desnutrición, resultando en un desequilibrio entre las necesidades corporales y la ingesta de nutrientes. Se hace referencia a los individuos con bajo peso como «malnutrición por déficit» y a los individuos con obesidad como «malnutrición por exceso»" En la actualidad México presenta un proceso de envejecimiento en ascenso; el Consejo Nacional de Población (CONAPO) calcula que para el año 2050 el $21.5 \%$ de la población general estará conformada por adultos de 65 años o más ${ }^{2}$. Esto representará un fuerte impacto a nivel social y económico del país, así como también en el tema de salud. Durante el proceso de envejecimiento ocurren alteraciones que provoca cambios en la estructura y función en diferentes órganos, lo que condiciona que esta población sea vulnerable al desarrollo de enfermedades crónicas $^{3,4}$ y por consiguiente presentan altas tasas de ingresos hospitalarios para el tratamiento de dichos padecimientos $^{5}$. Asimismo, los pacientes adultos mayores con bajo peso, sobrepeso u obesidad son más susceptibles a desarrollar complicaciones, que influyen negativamente en el tratamiento y pronóstico de estos pacientes ${ }^{6}$.

La desnutrición entre los pacientes hospitalizados con bajo peso u obesidad es significativamente más costosa para el sistema público de salud que el tratamiento de pacientes con un peso normal. Se estima que el costo del tratamiento de una persona con sobrepeso es un $10 \%$ más alto, mientras que el de una persona obesa es un $36 \%$ más alto que el de una persona con peso corporal normal. Además, la aparición de obesidad aumenta significativamente el riesgo de desarrollar complicaciones y mortalidad.

Los hospitales deben tener en cuenta el estado nutricional y la alimentación de los pacientes con bajo peso, así como con sobrepeso y obesidad, con el fin de proporcionar un tratamiento adecuado. La guía de práctica clínica para la evaluación y seguimiento nutricional del adulto mayor recomienda la aplicación del cuestionario Mini Nutritional Assessment (MNA), o evaluación mini-nutricional, para evaluar el estado nutricional en pacientes después de los 60 años 7 . Mediante el cuestionario MNA es posible predecir la aparición de malnutrición antes de que aparezcan alteraciones en las concentraciones de marcadores séricos. Además, permite una evaluación amplia del estado nutricional y la condición psicofísica del paciente ${ }^{8,9}$.

En un estudio de Fuchs, et al. se analizaron 561 pacientes adultos mayores del Hospital General de la Ciudad de México y se reportó que el 70\% de los pacientes presentó algún grado de desnutrición. Igualmente, se encontró que la deficiencia de los requerimientos energético-proteicos en los pacientes impidió la pronta recuperación y prolongó la estancia hospitalaria ${ }^{10}$.

México cuenta con poca información sobre el perfil de salud, nutrición y entorno del adulto mayor. En el 2001 se implementó la Encuesta Nacional de Salud y Envejecimiento (Enasem) en un esfuerzo de recolectar y brindar información de las dinámicas de salud de la población mexicana de edad avanzada ${ }^{11}$. Sin embargo, no se encuentran estudios realizados en Yucatán donde se examine el estado nutricional de pacientes adultos mayores que se encuentran hospitalizados después de ser sometidos a una cirugía. Debido a esto, el siguiente estudio se diseñó para evaluar el estado nutricional en pacientes hospitalizados utilizando el cuestionario MNA. Este estudio recibió la aprobación de los Comités de Investigación y Ética en Investigación del Hospital Regional de Alta Especialidad de la Península de Yucatán (HRAEPY). 


\section{MÉTODOS}

Se seleccionó una muestra aleatoria representativa de pacientes mayores de 60 años en periodo postoperatorio en el área de hospitalización, con base en las listas proporcionadas por el departamento de estadística del HRAEPY. Para el tamaño de la muestra se aplicó la fórmula para poblaciones infinitas; el tamaño mínimo necesario fue de 83 pacientes. Se evaluó a un total de 100 pacientes de ambos sexos intervenidos en el HRAEPY, los cuales fueron sometidos a cirugía de las diferentes especialidades, incluyendo angiología, cirugía general, coloproctología, neurocirugía, oftalmología, oncología y urología. La recolección de datos se realizó durante el periodo de febrero a mayo del 2019, previa firma de consentimiento informado. Se excluyeron aquellos pacientes que no aceptaran participar en el estudio.

Se aplicó el cuestionario MNA ${ }^{12}$, el cual consta de 18 preguntas divididas en cuatro bloques, los cuales a su vez constan de seis parámetros dietéticos, cuatro antropométricos, seis de evaluación global y dos de valoración subjetiva. Al sumar los puntajes se clasifica al paciente en las siguientes categorías: sin desnutrición (mayor de 23.5 puntos), en riesgo de malnutrición (de 17.5 a 23.5 puntos) y con malnutrición (menor de 17.5 puntos).

Las medidas antropométricas fueron evaluadas siguiendo las recomendaciones establecidas en la Guía para completar el formulario MNA ${ }^{12}$ y estuvo a cargo de una nutrióloga entrenada. El peso fue medido con una báscula de plataforma marca Detecto ${ }^{\circledR}$, calibrada en kilogramos, y para la talla un tallímetro, calibrado en centímetros. Para aquellos pacientes postoperatorios a quienes no se les pudo realizar la medición, se estimó la talla mediante la fórmula «altura de rodilla-talón» de Chumlea ${ }^{13}$. La altura de rodilla $(A R)$, circunferencia braquial $(C B)$ y circunferencia de pantorrilla (CP) fueron medidas con cinta métrica estándar, utilizado las referencias anatómicas establecidas. Se clasificó a los pacientes según su índice de masa corporal (IMC) modificado para el adulto mayor de acuerdo con la Guía de Práctica Clínica: Evaluación y Seguimiento Nutricional del Adulto Mayor ${ }^{7}$, en la cual se establece bajo peso si menor a $22 \mathrm{~kg} / \mathrm{m}^{2}$, peso normal de 22 a $27 \mathrm{~kg} / \mathrm{m}^{2}$, sobrepeso de 27.1 a $30 \mathrm{~kg} / \mathrm{m}^{2}$ y obesidad si mayor a $30 \mathrm{~kg} / \mathrm{m}^{2}$. Los estudios de laboratorio utilizados para evaluar el estado nutricional fueron: biometría hemática (hemoglobina, hematocrito, cuenta leucocitaria y cuenta plaquetaria), electrolitos séricos (sodio, potasio, cloro) y química sanguínea (glucosa, urea, creatinina, ácido úrico).

El estado nutricional de los adultos mayores se estimó por separado para hombres y mujeres. Todos los datos se expresan como promedio $(X)$, mediana (M) y desviación estándar (DE) para cada variable en cada grupo de participantes. Se estimó la normalidad de la distribución de las variables, cumpliendo con los criterios de la prueba de Shapiro-Wilk. Se realizó la prueba apropiada (t-test o $U$ de Mann-Whitney) para las diferencias significativas. Las diferencias entre los grupos femenino $(F)$ y masculino $(M)$ se realizaron utilizando el paquete estadístico Jamovi ${ }^{\circledR}$ (versión 0.9). Se consideró significativo un valor de $p<0.05$.

\section{RESULTADOS}

La población estuvo compuesta por 100 pacientes del HRAEPY, $55 \%$ del sexo femenino y $45 \%$ del sexo masculino. La media de edad fue 68 años para ambos grupos, con una edad mínima de 60 y una máxima de 93 años. El peso fue mayor en el grupo masculino $(71.8 \pm 13.58)$ que en el grupo femenino $(62.4 \pm 14.28)$. El promedio del IMC fue de 29.71 y 28.02 en pacientes femeninos y masculinos, respectivamente. Se observó en pacientes masculinos presiones arteriales sistólicas (PAS) y diastólicas (PAD) elevadas (PAS: $121.96 \pm 17.94$; PAD: $72.96 \pm 18.53)$ en comparación con el grupo de mujeres (PAS: $118.36 \pm$ 19.72; PAD: $71.87 \pm 12.70$ ). El grupo femenino mostró resultados de leucocitos mayores $(10.72 \pm 6.34)$ que el grupo masculino $(7.71 \pm 6.58)$. En cuanto a la glucosa, el grupo femenino tuvo un promedio de 140.38 y el grupo masculino de 117.74 (Tabla 1).

El $58.18 \%$ de las mujeres manifestaron tener pérdida de peso en los últimos tres meses; el promedio de pérdida de peso en mujeres fue del $4.6 \%(3.04 \mathrm{~kg})$ y en hombres del $4.3 \%(3.36 \mathrm{~kg})$. Solo 16 pacientes (8 mujeres y 8 hombres) tuvieron una pérdida de peso mayor del 10\%. El promedio de días de estancia fue de 3.86 días en pacientes con estado nutricional normal, 10.4 con riesgo de malnutrición y 9.88 con malnutrición. El $47 \%$ de las mujeres y el $40 \%$ de los hombres tuvieron un IMC > $23 \mathrm{~kg} / \mathrm{m}^{2}$. El estado nutricional prevalente, valorado por la escala de IMC en pacientes adultos mayores, fue el peso normal (30\%), seguido de obesidad tipo 1 (26\%) y sobrepeso (19\%). En relación con el IMC, del $16 \%$ de los pacientes con estado nutricional 
Tabla 1. Características del estudio de población según el sexo

\begin{tabular}{|c|c|c|c|c|c|}
\hline \multirow[t]{2}{*}{ Parámetros } & \multicolumn{2}{|c|}{$\begin{array}{l}\text { Sexo femenino } \\
\quad(n=55)\end{array}$} & \multicolumn{2}{|c|}{$\begin{array}{l}\text { Sexo masculino } \\
\quad(n=45)\end{array}$} & \multirow[t]{2}{*}{ Valor de $p$} \\
\hline & $\mathrm{X} \pm \mathrm{DE}$ & M & $X \pm D E$ & M & \\
\hline Edad (años) & $69.10 \pm 6.09$ & 68 & $68.8 \pm 7.36$ & 68 & 0.625 \\
\hline Altura $(\mathrm{cm})$ & $145.50 \pm 7.05$ & 144.86 & $158.76 \pm 8.02$ & 156.61 & 0.099 \\
\hline Peso (kg) & $62.4 \pm 14.28$ & 62.0 & $71.8 \pm 13.58$ & 71.00 & $0.001^{*}$ \\
\hline Índice de masa corporal & $29.63 \pm 6.96$ & 29.71 & $28.46 \pm 4.80$ & 28.02 & 0.715 \\
\hline Circunferencia de brazo (cm) & $27.12 \pm 3.91$ & 27 & $28.8 \pm 3.60$ & 29 & 0.325 \\
\hline Circunferencia de pantorrilla (cm) & $31.29 \pm 4.11$ & 31 & $33.06 \pm 3.96$ & 33 & 0.185 \\
\hline Altura de rodilla $(\mathrm{cm})$ & $42.13 \pm 3.69$ & 42 & $48.18 \pm 3.99$ & 47 & 0.085 \\
\hline PAS (mmHg) & $118.36 \pm 19.72$ & 120 & $121.96 \pm 17.94$ & 120 & 0.316 \\
\hline PAD $(\mathrm{mmHg})$ & $71.87 \pm 12.70$ & 70 & $72.69 \pm 18.53$ & 70 & 0.969 \\
\hline Hemoglobina & $11.18 \pm 1.61$ & 11.3 & $11.38 \pm 2.22$ & 10.9 & 0.909 \\
\hline Hematocrito & $34.26 \pm 4.72$ & 34.4 & $35.05 \pm 6.40$ & 33.9 & 0.835 \\
\hline Leucocitos & $10.72 \pm 6.34$ & 9.09 & $7.71 \pm 6.58$ & 9.43 & 0.819 \\
\hline Neutrófilos totales & $7.71 \pm 6.58$ & 6 & $8.25 \pm 7.23$ & 6.23 & 0.41 \\
\hline Linfocitos totales & $1.64 \pm 0.80$ & 1.54 & $1.47 \pm 0.79$ & 1.28 & $0.004^{*}$ \\
\hline Plaquetas & $285.32 \pm 131.93$ & 271 & $271.72 \pm 162.62$ & 246 & 0.436 \\
\hline Sodio & $140.59 \pm 15.56$ & 138 & $138 \pm 5.41$ & 139 & 0.887 \\
\hline Potasio & $4.01 \pm 0.5$ & 4 & $4.16 \pm 0.63$ & 4.1 & 0.439 \\
\hline Cloro & $107.72 \pm 15.05$ & 107 & $103.07 \pm 15.67$ & 106 & 0.314 \\
\hline Glucosa & $140.38 \pm 103.58$ & 101 & $117.74 \pm 47.26$ & 103.1 & 0.967 \\
\hline Urea & $41.59 \pm 88.5$ & 24 & $41.87 \pm 52.59$ & 28 & 0.383 \\
\hline Creatinina & $0.88 \pm 0.45$ & 0.76 & $1.14 \pm 1.32$ & 0.91 & 0.103 \\
\hline Ácido úrico & $4.79 \pm 1.93$ & 4.6 & $4.62 \pm 2.11$ & 4.5 & 0.382 \\
\hline
\end{tabular}

${ }^{*} p<0.05$.

n: número de personas; $\mathrm{X} \pm \mathrm{DE}$ : promedio \pm desviación estándar; M: media; PAS: presión arterial sistólica; PAD: presión arterial diastólica.

normal, el $37.5 \%$ se clasificaron en normopeso y $50 \%$ en obesidad; del 53\% con riesgo de malnutrición, el $26.6 \%$ se clasificaron con sobrepeso, el $47.2 \%$ con obesidad y el $28.3 \%$ con normopeso. Del $31 \%$ de los pacientes con malnutrición, el $22.6 \%$ con peso bajo, el $29 \%$ se clasificaron con normopeso y con obesidad, respectivamente (Tabla 2 ).

Al estratificar a los pacientes de acuerdo con el tipo de cirugía, se encontró con riesgo de malnutrición al 37.7\% de los sometidos a cirugía general y al $20.7 \%$ de los sometidos a oncocirugía. Presentaron malnutrición el $45.2 \%$ de cirugía general, el $16.1 \%$ de oncocirugía y el $12.9 \%$ de urología y angiología, respectivamente.

Por puntaje de la evaluación del MNA, el 52\% tenían riesgo de malnutrición, con un puntaje promedio de 20.84, y con mayor prevalencia en el sexo femenino
(31\% femenino vs. 21\% masculino), 26\% malnutrición (13\% femenino y $13 \%$ masculino) y el $22 \%$ estado nutricional normal (11\% femenino y $11 \%$ masculino) (Fig. 1).

En cuanto al grado de nutrición en los pacientes que vivían solos (63\%) se encontró que el $22.2 \%$ tenían estado nutricional normal, el 58.7\% tenían riesgo de malnutrición y el 19\% malnutrición. Por otro lado, el 37\% de los pacientes no vivían solos; de estos, el $21.6 \%$ tenían estado nutricional normal, el 40.5\% tenían riesgo de malnutrición y el 37.8\% tenían malnutrición (Tabla 4).

El tipo de alimentación indicada más frecuente fue la enteral, con 68\%; de estos pacientes el $72.05 \%$ presentaba dentadura incompleta, el $20.58 \%$ dentadura postiza y solo el $7.35 \%$ tenían dentadura completa. Los pacientes en ayuno representaron el $27 \%$, de los cuales el $40.7 \%$ tenían dentadura incompleta, el 33.3\% dentadura postiza 
Tabla 2. Relación del índice de masa corporal y el estado nutricional de adultos mayores en el postoperatorio

\begin{tabular}{lccc}
\hline & $\begin{array}{c}\text { Estado nutricional normal } \\
(\mathbf{n}=\mathbf{1 6})\end{array}$ & $\begin{array}{c}\text { Riesgo de malnutrición } \\
(\mathbf{n}=\mathbf{5 3})\end{array}$ & $\begin{array}{c}\text { Malnutrición } \\
(\mathbf{n = 3 1 )}\end{array}$ \\
\hline Peso bajo & $1(6.2 \%)$ & $1(1.9 \%)$ & $7(22.6 \%)$ \\
Normopeso & $6(37.5 \%)$ & $15(28.3 \%)$ & $9(29.0 \%)$ \\
Sobrepreso & $1(6.2 \%)$ & $12(22.6 \%)$ & $6(19.3 \%)$ \\
Obesidad & $8(50.0 \%)$ & $25(47.2 \%)$ & $9(29.0 \%)$ \\
\hline
\end{tabular}

Tabla 3. Relación entre el tipo de cirugía y el estado nutricional de adultos mayores

\begin{tabular}{lccc}
\hline & $\begin{array}{c}\text { Estado nutricional normal } \\
(\mathbf{n = 1 6 )}\end{array}$ & $\begin{array}{c}\text { Riesgo de malnutrición } \\
(\mathbf{n}=\mathbf{5 3})\end{array}$ & $\begin{array}{c}\text { Malnutrición } \\
(\mathbf{n}=\mathbf{3 1})\end{array}$ \\
\hline Angiología & $1(6.2 \%)$ & $8(15.0 \%)$ & $4(12.9 \%)$ \\
Cirugía general & $6(37.5 \%)$ & $20(37.7 \%)$ & $14(45.2 \%)$ \\
Coloproctología & 0 & $4(7.5 \%)$ & 0 \\
Neurocirugía & 0 & $2(3.8 \%)$ & $2(6.5 \%)$ \\
Oftalmología & $3(18.8 \%)$ & $3(5.6 \%)$ & $2(6.5 \%)$ \\
Oncocirugía & 0 & $5(9.4 \%)$ & $5(16.1 \%)$ \\
Urología & $6(37.5 \%)$ & $11(20.7 \%)$ & $4(12.9 \%)$ \\
\hline
\end{tabular}

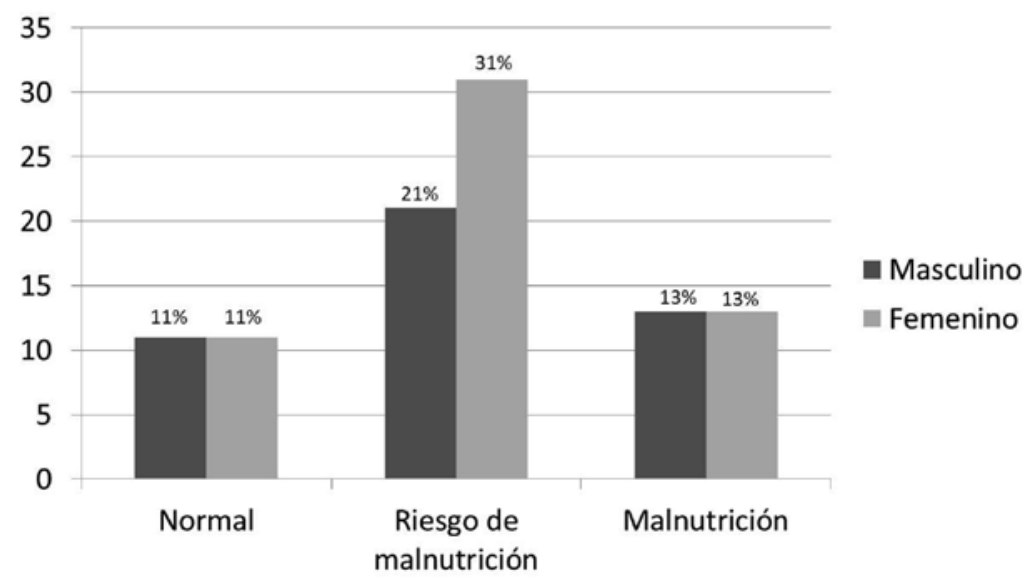

Figura 1. Estado nutricional en adultos mayores en el postoperatorio.

y el $25.9 \%$ dentadura completa. La nutrición parenteral se presentó solo en el $5 \%$ de los pacientes, y de estos el $80 \%$ tenía dentadura incompleta, el 20\% dentadura postiza y ningún paciente presentó dentadura completa.

\section{DISCUSIÓN}

Los resultados de este estudio indican que más de la mitad de los adultos mayores hospitalizados se encontraron en riesgo de malnutrición (53\%). Este porcentaje corresponde a lo reportado en otros estudios en adultos mayores en México. Gutiérrez Reyes, et al. reportaron un $69 \%$ de riesgo de malnutrición entre los adultos mayores al ingreso hospitalario en la Ciudad de México55; asimismo, Osuna Padilla, et al. encontraron un $47.2 \%$ en riesgo de malnutrición en usuarios y residentes de distintos centros de asistencia social en Sinaloa ${ }^{14}$. Es importante señalar que en nuestro estudio se hallaron frecuencias elevadas de malnutrición (31\%), en comparación a lo encontrado en otros estudios en poblaciones similares, en donde las frecuencias varían entre el 18.6 y el $16.5 \%$.

Se observó que la mayoría de los participantes de la investigación tenían sobrepeso u obesidad. En las últimas décadas, el perfil epidemiológico ha cambiado entre los adultos mayores en México, pasando desde la desnutrición a la obesidad. En este estudio se 
Tabla 4. Valores promedio y valores anormales de los parámetros analizados, basados en un cuestionario MNA, en una población de estudio de hombres y mujeres

\begin{tabular}{|c|c|c|c|c|c|}
\hline \multirow{2}{*}{$\begin{array}{l}\text { Mini Nutritional Assessment } \\
\text { Parámetros antropométricos }\end{array}$} & \multicolumn{2}{|c|}{$\begin{array}{l}\text { Sexo femenino } \\
\quad(n=55)\end{array}$} & \multicolumn{2}{|c|}{$\begin{array}{l}\text { Sexo masculino } \\
\quad(n=45)\end{array}$} & \multirow[t]{2}{*}{ Valor de $\mathrm{p}$} \\
\hline & $X \pm S D$ & n (\%) & $X \pm S D$ & $\mathrm{n}(\%)$ & \\
\hline $\mathrm{IMC}>23 \mathrm{~kg} / \mathrm{m}^{2}$ & $31.3 \pm 5.67$ & 47 & $29.4 \pm 4.26$ & 40 & 0.111 \\
\hline $\mathrm{CB}>21 \mathrm{~cm}$ & $27.3 \pm 3.74$ & 54 & $28.8 \pm 3.6$ & 45 & 0.054 \\
\hline $\mathrm{CP}>31 \mathrm{~cm}$ & $34 \pm 2.73$ & 33 & $34.4 \pm 3.06$ & 36 & 0.439 \\
\hline$\underline{A R}$ & $42.1 \pm 3.7$ & 55 & $48.2 \pm 4$ & 45 & 0.085 \\
\hline \multirow[t]{2}{*}{ Pérdida de peso en los últimos 3 meses } & \multicolumn{2}{|c|}{ n (\%) } & \multicolumn{2}{|c|}{ n (\%) } & \\
\hline & \multicolumn{2}{|c|}{$32(58.18)$} & \multicolumn{2}{|c|}{$28(62.22)$} & \\
\hline Nutrición & \multicolumn{2}{|c|}{ n (\%) } & \multicolumn{2}{|c|}{ n (\%) } & \\
\hline$<3$ comidas al día & \multicolumn{2}{|c|}{$12(21.81)$} & \multicolumn{2}{|c|}{$10(22.22)$} & \\
\hline $\begin{array}{l}\text { Sin ingesta diaria de productos lácteos, carne, } \\
\text { pescado o aves y alimentos que contengan huevos } \\
\text { o legumbres por semana }\end{array}$ & \multicolumn{2}{|c|}{$22(40.00)$} & \multicolumn{2}{|c|}{$12(26.66)$} & \\
\hline Frutas y verduras $<2$ piezas/día & \multicolumn{2}{|c|}{$19(39.54)$} & \multicolumn{2}{|c|}{$19(42.22)$} & \\
\hline Pacientes con disminución del apetito & \multicolumn{2}{|c|}{$33(60.00)$} & \multicolumn{2}{|c|}{$22(48.88)$} & \\
\hline$<5$ vasos de agua/día & \multicolumn{2}{|c|}{$27(49.09)$} & \multicolumn{2}{|c|}{$22(48.88)$} & \\
\hline $\begin{array}{l}\text { Paciente incapaz de comer solo o come solo con } \\
\text { dificultad }\end{array}$ & \multicolumn{2}{|c|}{$18(32.72)$} & \multicolumn{2}{|c|}{$14(31.11)$} & \\
\hline Autopercepción del estado nutricional & \multicolumn{2}{|c|}{ n (\%) } & \multicolumn{2}{|c|}{ n (\%) } & \\
\hline \multicolumn{6}{|l|}{ El paciente se ve a sí mismo: } \\
\hline - Malnutrido grave & \multicolumn{2}{|c|}{$7(12.72)$} & \multicolumn{2}{|c|}{$5(11.11)$} & \\
\hline - No lo sabe o malnutrición moderada & \multicolumn{2}{|c|}{$25(45.45)$} & \multicolumn{2}{|c|}{$17(37.77)$} & \\
\hline - Sin problemas de nutrición & 23 & & & & \\
\hline $\begin{array}{l}\text { En comparación con personas de su edad, el } \\
\text { paciente considera su estado de salud: }\end{array}$ & & & & & \\
\hline - Peor & 13 & & & & \\
\hline - No lo sabe & & & & & \\
\hline - Igual & 22 & & & & \\
\hline - Mejor & 14 & & & & \\
\hline Evaluación general & & & & & \\
\hline Vive independientemente en su domicilio & & & & & \\
\hline Toma más de 3 medicamentos al día & 25 & & & & \\
\hline $\begin{array}{l}\text { Ha tenido una enfermedad aguda o situación de } \\
\text { estrés psicológicos en los últimos } 3 \text { meses }\end{array}$ & & & & & \\
\hline EL paciente tiene movilidad sin problemas & 20 & & & & \\
\hline Sin problemas neuropsicológicos & 30 & & & & \\
\hline Úlceras o lesiones cutáneas & 18 & & & & \\
\hline
\end{tabular}

IMC: índice de masa corporal; CB: circunferencia braquial; CP: circunferencia de pantorrilla; AR: altura de rodilla.

observó que la malnutrición se relacionó no solamente con bajo peso, sino también con sobrepeso y obesidad. Datos obtenidos en este estudio describen en adultos mayores hospitalizados un IMC $>23 \mathrm{~kg} / \mathrm{m}^{2}$ promedio de $30.48 \mathrm{~kg} / \mathrm{m}^{2}$, una $C B>21 \mathrm{~cm}$ de $27.64 \mathrm{~cm}$, una
$\mathrm{CP}>31 \mathrm{~cm}$ promedio de $34.19 \mathrm{~cm}$ y una AR de 45.01 $\mathrm{cm}$, cifras muy elevadas con respecto a las observadas en la población sana del mismo grupo etario ${ }^{14}$. El aumento en las frecuencias de la obesidad conlleva el empeoramiento del curso y el tratamiento de la 
enfermedad, lo que resulta no solo en una hospitalización prolongada, sino que también afecta el estado nutricional del paciente ${ }^{15}$

Actualmente se pueden encontrar diversos instrumentos para la detección de pacientes con malnutrición; sin embargo, algunos son muy extensos o de difícil aplicación. El instrumento MNA ha demostrado aplicabilidad en diversas poblaciones, además de que supone la reducción del tiempo de estancia intrahospitalaria ${ }^{8}$. En un estudio realizado por Slee, et al. en 78 pacientes adultos mayores hospitalizados del Reino Unido, en donde se compararon la herramienta universal para detección de malnutrición por déficit (MUST, por sus siglas en inglés) y el MNA, se encontró en este último instrumento gran precisión para la clasificación y diagnóstico correcto de pacientes de este grupo etario en riesgo de malnutrición, evitando sesgos durante la evaluación ${ }^{16}$. El reconocimiento temprano de la desnutrición y la intervención adecuada en pacientes hospitalizados pueden prevenir complicaciones posteriores en el tratamiento y la mejoría de estos pacientes. Este estudio es el primer reporte del estado nutricio de la población adulta mayor en Yucatán en el periodo postoperatorio, y beneficia potencialmente al equipo del área de la salud conformado por nutricionistas, médicos, enfermeros, entre otros, con la finalidad de concienciarlos en el manejo y la adecuada clasificación del estado nutricional del paciente adulto mayor y así evitar comorbilidades.

\section{CONCLUSIÓN}

La malnutrición y el riesgo de malnutrición fueron hallazgos frecuentes entre los adultos mayores que acuden al hospital. En este estudio la aplicación del cuestionario MNA proporcionó resultados fiables y que pueden ser fácilmente interpretados por médicos y profesionales de la salud, lo que resultaría en detección temprana de amenazas relacionadas con la desnutrición entre los adultos mayores en Yucatán.

\section{CONTRIBUCIONES DE LOS AUTORES}

Los autores Rodrigo García-Pérez, Sarahí Herrera-Escalante y Jocelyne de la Cruz-Acosta han contribuido de igual forma a la elaboración de este artículo.

\section{BIBLIOGRAFÍA}

1. Ravasco P, Anderson H, Mardones F. Métodos de valoración del estado nutricional. Nutr Hosp. 2010;25:57-66.

2. González K. Envejecimiento demográfico en México: análisis comparativo entre las entidades federativas. En: La situación demográfica de México en 2015. México: Consejo Nacional de Población; 2015. pp.113-29.

3. Socarrás Suárez MM, Bolet Astoviza M, Castañeda Abascall, Betancourt Baltrell D. Desnutrición de los pacientes de la tercera edad ingresados en el Hospital "General Calixto García”. Rev Cubana Invest Biomed. 2006;25(4).

4. González-González C, Palloni A, Wong R. Mortality and its association with chronic and infectious diseases in Mexico: A panel data analysis of the elderly. Salud Publica Mex. 2015;57:s39-45.

5. Gutiérrez Reyes J, Serralde Zúñiga A, Guevara Cruz MJNH. Prevalencia de desnutrición del adulto mayor al ingreso hospitalario. Nutr Hosp. 2007;22(6):702-9.

6. Ortiz Saavedra PJ, Méndez Silva FJ, Varela Pinedo L, Pamo Reyna 0. Variación del estado nutricional del paciente adulto mayor durante la hospitalización en los servicios de medicina de un hospital general. Rev Med Hered. 2007;18(1):4-9.

7. Evaluación y seguimiento nutricional del adulto mayor en el primer nivel de atención [Internet]. México: Consejo de Salubridad General; 2014. Disponible en: http://www.cenetec.salud.gob.mx/descargas/gpc/CatalogoMaestro/095 GPC_Evaycontrolnutic_eneladultomayor/NUTRICION_AM_RR_CENETEC.pdf

8. Christner S, Ritt M, Volkert D, Wirth R, Sieber C, Gaßmann KG. Evaluation of the nutritional status of older hospitalised geriatric patients: a comparative analysis of a Mini Nutritional Assessment (MNA) version and the Nutritional Risk Screening (NRS 2002). J Hum Nutr Diet. 2016;29(6): 704-13.

9. Izaola 0, Román L, Cabezas G, Rojo S, Cuellar L, Terroba M, et al. Mini Nutritional Assessment (MNA) como método de evaluación nutricional en pacientes hospitalizados. An Med Inter. 2005:22(7):313-6.

10. Fuchs V, Mostkoff D, Gutiérrez Salmeán G, Amancio OJNH. Estado nutricio en pacientes internados en un hospital público de la Ciudad de México. Nutr Hosp. 2008;23(3):294-303.

11. Puig A, Pagán JA, Soldo BJ. Envejecimiento, salud y economía: la Encuesta Nacional sobre Salud y Envejecimiento en México. Trimest Econ. 2006;73(290):407-18.

12. Guía para rellenar el formulario Mini Nutritional Assessment (MNA) [Internet]. Nestlé Nutrition Institute. Disponible en: https://www.mna-elderly.com/ forms/mna_guide_spanish.pdf

13. Guzmán Hernández C, Reinoza Calderón G, Hernández Hernández R. Estimación de la estatura a partir de la longitud de pierna medida con cinta métrica. Nutr Hosp. 2005;20(5):358-63.

14. Osuna-Padilla IA, Verdugo-Hernández S, Leal-Escobar G, Osuna-Ramírez I. Estado nutricional en adultos mayores mexicanos: estudio comparativo entre grupos con distinta asistencia social. Rev Esp Nutr Hum Diet. 2015;19(1):12-20.

15. Penié JB, Porbén SS, González CM. Programa de intervención alimentaria, nutrimental y metabólica del paciente hospitalizado. Rev Cubana Aliment Nutr. 1999;13(2):137-44.

16. Slee A, Birch D, Stokoe D. A comparison of the malnutrition screening tools, MUST, MNA and bioelectrical impedance assessment in frail older hospital patients. Clin Nutr. 2015;34(2):296-301. 